\title{
Quadrupolarizability of Liquid Mixtures
}

\author{
Supporting Information
}

\author{
Iglika M. Dimitrova*1,2, Velislava I. Yordanova ${ }^{1}$, Radomir I. Slavchov ${ }^{3}$ \\ ${ }^{1}$ University of Chemical Technology and Metallurgy, Department of Physical Chemistry, \\ 8, Kliment Ohridski Blvd., BG-1756 Sofia, Bulgaria \\ ${ }^{2}$ Institute of Solid State Physics, Bulgarian Academy of Sciences \\ 72 Tzarigradsko Chaussee Blvd., BG-1784 Sofia, Bulgaria \\ ${ }^{3}$ School of Engineering and Materials Science, Queen Mary University of London, \\ Department of Chemical Engineering, Mile End Road, London E1 4NS, UK
}

\section{S1. List of Symbols}

$C \quad$ particle density $\left[\mathrm{m}^{-3}\right]$

$\boldsymbol{E}_{0} \quad$ external electric field $\left[\mathrm{V} \mathrm{m}^{-1}\right]$

$\boldsymbol{E}_{\text {cav }}$ cavity electric field [ $\left.\mathrm{V} \mathrm{m}^{-1}\right]$

$\boldsymbol{E}_{\text {react }}$ reaction electric field $\left[\mathrm{V} \mathrm{m}^{-1}\right]$

$\nabla \boldsymbol{E}_{0} \quad$ external field gradient $\left[\mathrm{V} \mathrm{m}^{-2}\right]$

$f_{p}, f_{q}, f_{E}, f_{\nabla E}, g_{p}, g_{q} \quad$ quadrupole factors [/]

$k_{\mathrm{B}} \quad$ Boltzmann constant $[\mathrm{J} / \mathrm{K}]$

$k_{0}, k_{\rho}$ coefficients in the empirical dependence Eq. (17) between density and cavity radius; $k_{0}\left[\mathrm{~kg} / \mathrm{m}^{3}\right], k_{\rho}[/]$

$L_{Q} \quad$ quadrupolar length of the medium [m]

$m$ mass of a molecule [kg]

$\boldsymbol{P} \quad$ polarization of the medium $\left[\mathrm{C} \mathrm{m}^{-2}\right]$

$\boldsymbol{p} \quad$ dipole moment of a molecule in external field $[\mathrm{C} \mathrm{m}]$

$\boldsymbol{p}_{0} \quad$ permanent (intrinsic) dipole moment of a molecule [C $\left.\mathrm{m}\right]$

$\boldsymbol{Q}$ quadrupolarization of the medium $\left[\mathrm{C} \mathrm{m}^{-1}\right]$

$\boldsymbol{q}$ quadrupole moment of a molecule in external field gradient $\left[\mathrm{C} \mathrm{m}^{2}\right]$

$\boldsymbol{q}_{0} \quad$ permanent (intrinsic) quadrupole moment of a molecule $\left[\mathrm{C} \mathrm{m}^{2}\right]$

$R_{\text {cav }} \quad$ radius of the cavity [m]

$T$ absolute temperature $[\mathrm{K}]$

U unit tensor

$v_{i} \quad$ partial molecular volume $\left[\mathrm{m}^{3}\right]$

$X_{p} \quad$ reaction field factor $\left[\mathrm{F}^{-1} \mathrm{~m}^{-2}\right]$

$X_{q} \quad$ reaction field gradient factor $\left[\mathrm{F}^{-1} \mathrm{~m}^{-4}\right]$

$x=L_{Q} / R_{\text {cav }}[/]$

$y_{i} \quad$ mole fraction of $i$-th component [/]

$Y_{E} \quad$ cavity field factor [/]

$Y_{\nabla E} \quad$ cavity field gradient factor [/]

$\alpha_{p} \quad$ mean polarizability of a molecule [ $\left.\mathrm{F} \mathrm{m}^{2}\right]$

$\alpha_{P} \quad$ macroscopic polarizability of the medium $\left[\mathrm{F} \mathrm{m}^{-1}\right]$

$\alpha_{q} \quad$ mean quadrupolarizability of a molecule [ $\left.\mathrm{F} \mathrm{m}^{4}\right]$

$\alpha_{Q} \quad$ macroscopic quadrupolarizability of the medium [F m]

$\varepsilon \quad$ absolute dielectric permittivity of the medium [F m$\left.{ }^{-1}\right]$ 
$\varepsilon_{0} \quad$ dielectric permittivity of the vacuum $\left[\mathrm{F} \mathrm{m}^{-1}\right]$

$\phi \quad$ electrostatic potential [V]

$\rho \quad$ mass density $\left[\mathrm{kg} \mathrm{m}^{-3}\right]$

$\rho_{\mathrm{el}} \quad$ free charge number density $\left[\mathrm{C} \mathrm{m}^{-3}\right]$

$\rho_{i}=m_{i} / v_{i}\left[\mathrm{~kg} \mathrm{~m}^{-3}\right]$

\section{S2. Pure Component Quadrupolar Lengths}

We supplement here for comparison the pure component quadrupolar lengths of methane and nitrogen liquids as calculated in Ref. $[9,10]$. As one can see, the quadrupolar length of methane does not depend on temperature (Figure S1a) because methane does not possess intrinsic quadrupole moment and $\boldsymbol{q}_{0, \mathrm{CH}_{4}}: \boldsymbol{q}_{0, \mathrm{CH}_{4}} / k_{\mathrm{B}} T=0$ (as follows from Eq. (8) for pure methane). On the other hand, the points for nitrogen are dispersed (Figure S1b) due to the variation in temperature (indicated by the colour) producing respective variation of the macroscopic quadrupolarizability through the non-zero term $\boldsymbol{q}_{0, \mathrm{~N}_{2}}: \boldsymbol{q}_{0, \mathrm{~N}_{2}} / k_{\mathrm{B}} T$.
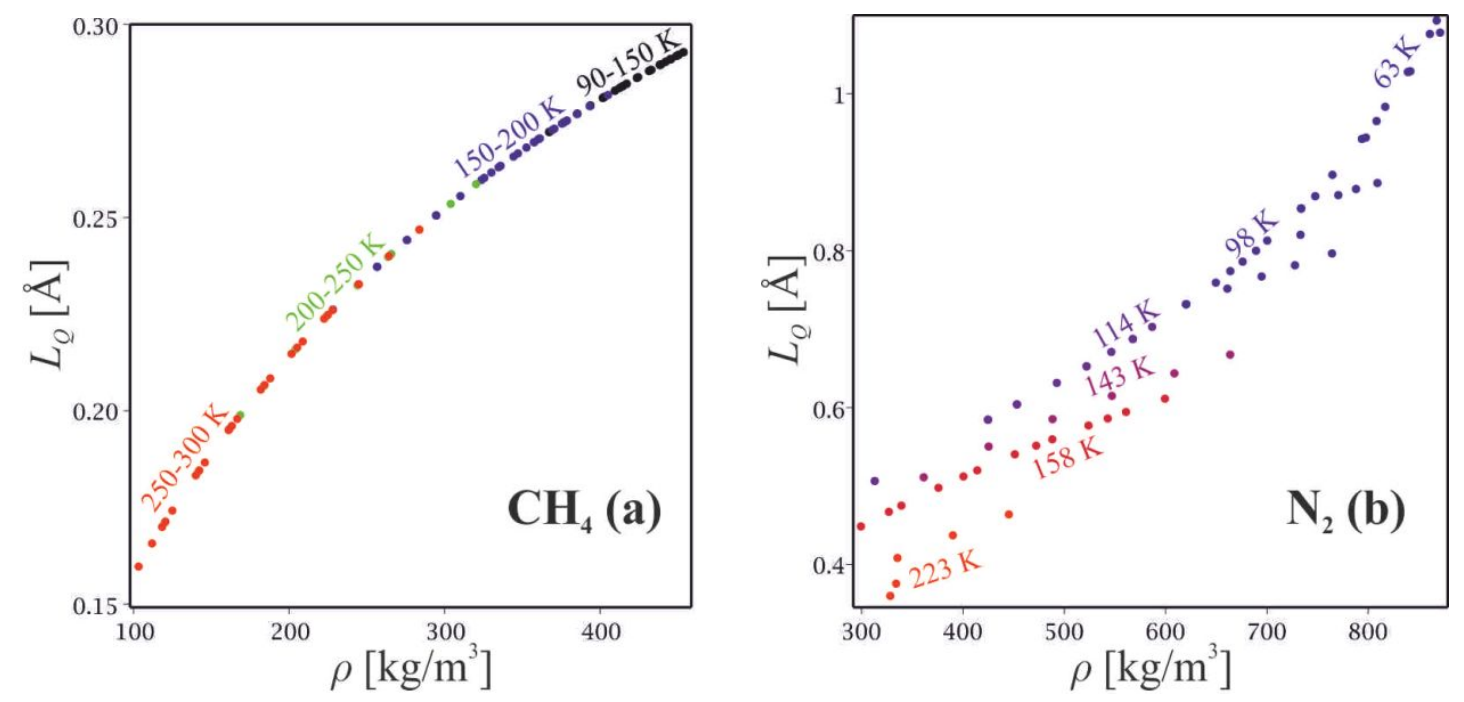

Figure S1. Pure component quadrupolar lengths $L_{Q}$ of methane $\mathrm{CH}_{4}$ (a) and nitrogen $\mathrm{N}_{2}$ (b) liquids as function of mass density $\rho$.

\section{S3. Calculation of the Molar Volumes of the Components}

According to Hankinson-Thomson technique ${ }^{29,30}$ the compressed liquid molar volume of a mixture is given by the Tait equation:

$$
V=V_{s m}\left(1-C \ln \frac{B+p}{B+P_{s m}}\right),
$$

where the volume is in $\left[\mathrm{m}^{3}\right]$ and the pressure is in $[\mathrm{Pa}]$. The coefficients $B$ and $C$ are

$$
\begin{gathered}
B / P_{c m}=-1+a_{1}\left(1-T_{R m}\right)^{1 / 3}+b_{1}\left(1-T_{R m}\right)^{2 / 3}+d_{1}\left(1-T_{R m}\right)+e_{1}\left(1-T_{R m}\right)^{4 / 3}, \\
C=j_{1}+k_{1} \omega_{\mathrm{SRK} m} .
\end{gathered}
$$


Here the coefficient $e_{1}$ is given by

$$
e_{1}=\exp \left(f_{1}+g_{1} \omega_{\mathrm{SRK} m}+h_{1} \omega_{\mathrm{SRK} m}^{2}\right)
$$

and all other constants are summarized in Table S1. The saturated volume of the mixture $V_{s m}$ is

$$
V_{s m}=V_{m}^{*} V_{R}^{(0)}\left[1-\omega_{\mathrm{SRK} m} V_{R}^{(\delta)}\right],
$$

where

$$
\begin{gathered}
V_{R}^{(0)}=1+a_{2}\left(1-T_{R m}\right)^{1 / 3}+b_{2}\left(1-T_{R m}\right)^{2 / 3}+c_{2}\left(1-T_{R m}\right)+d_{2}\left(1-T_{R m}\right)^{4 / 3}, \\
V_{R}^{(\delta)}=\left[e_{2}+f_{2} T_{R m}+g_{2} T_{R m}^{2}+h_{2} T_{R m}^{3}\right] /\left(T_{R m}-1.00001\right) .
\end{gathered}
$$

Table S1. Values of the constants in Eqs. (S.2)-(S.7).

\begin{tabular}{cc}
\hline \hline Constants in Eqs. (S.2), (S.3) and (S.4) & Constants in Eqs. (S.6) and (S.7) \\
\hline$a_{1}=-9.070217$ & $a_{2}=-1.52816$ \\
\hline$b_{1}=62.45326$ & $b_{2}=1.43907$ \\
\hline$d_{1}=-135.1102$ & $c_{2}=-0.81446$ \\
\hline$f_{1}=4.79594$ & $d_{2}=0.190454$ \\
\hline$g_{1}=0.250047$ & $e_{2}=-0.296123$ \\
\hline$h_{1}=1.14188$ & $f_{2}=0.386914$ \\
\hline$j_{1}=0.0861488$ & $g_{2}=-0.0427258$ \\
\hline$k_{1}=0.0344483$ & $h_{2}=-0.0480645$ \\
\hline \hline
\end{tabular}

The saturated pressure of the mixture $P_{s m}$ is calculated by the relation

$$
P_{s m}=P_{c m} P_{R m} .
$$

Here the critical pressure $P_{c m}$ is

$$
P_{c m}=\left(Z_{c m} R T_{c m}\right) / V_{m}^{*}
$$

and the compressibility factor $Z_{c m}$ is calculated using the following expression

$$
Z_{c m}=0.291-0.080 \omega_{\mathrm{SRK} m} \text {. }
$$

The reduced pressure of the mixture $P_{R m}$ is given by

$$
\log P_{R m}=P_{R m}^{(0)}+\omega_{\mathrm{SRK} m} P_{R m}^{(1)},
$$

where

$$
\begin{gathered}
P_{R m}^{(0)}=5.8031817 \log T_{R m}+0.07608141 \alpha, \\
P_{R m}^{(1)}=4.86601 \beta, \\
\alpha=35.0-36.0 / T_{R m}-96.736 \log T_{R m}+T_{R m}^{6}, \\
\beta=\log T_{R m}+0.03721754 \alpha .
\end{gathered}
$$

Reduced temperature $T_{R m}$ is

$$
T_{R m}=T / T_{c m} .
$$

Hankinson, Brobst, and Thomson propose the following mixing rules:

$$
\begin{gathered}
T_{c m}=\frac{\sum_{i} \sum_{j} y_{i} y_{j}\left(V_{i}^{*} T_{c i} V_{j}^{*} T_{c j}\right)^{1 / 2}}{V_{m}^{*}}, \\
V_{m}^{*}=\frac{1}{4}\left[\sum_{i} y_{i} V_{i}^{*}+3\left(\sum_{i} y_{i} V_{i}^{* 2 / 3}\right)\left(\sum_{i} y_{i} V_{i}^{* 1 / 3}\right)\right],
\end{gathered}
$$

and

$$
\omega_{\mathrm{SRK} m}=\sum_{i} y_{i} \omega_{\mathrm{SRK} i} .
$$


In these equations $y_{i}$ is the component mole fraction, $T_{c i}$ and $V_{i}^{*}$ are the pure component parameters which are given for methane and nitrogen in Table S2 (for other compounds see Table 3-10, p. 57 in Ref. [30]).

Table S2. Pure component parameters for the Hankinson-Brobst-Thomson model.

\begin{tabular}{lccc}
\hline \hline & $T_{c}$ & $\omega_{\text {SRK }}$ & $V^{*}$ \\
& {$[\mathrm{~K}]$} & {$[/]$} & {$[\mathrm{L} / \mathrm{mol}]$} \\
\hline $\mathrm{CH}_{4}$ & 190.58 & 0.0074 & 0.0994 \\
\hline $\mathrm{N}_{2}$ & 126.25 & 0.0358 & 0.0901 \\
\hline \hline
\end{tabular}

Substituting Eqs. (S.2)-(S.19) and the parameters from Table S1 and Table S2 in Eq. (S.1) yields liquid mixture volume $V$ as a function of the composition (for given $T$ and $p$ ). Then for two-component mixture we can calculate the molar volume of the components by

$$
v_{1}=V+y_{2}\left(\frac{\partial V}{\partial y_{1}}\right)_{y_{2}}
$$

and

$$
v_{2}=V-y_{1}\left(\frac{\partial V}{\partial y_{1}}\right)_{y_{2}},
$$

where $y_{2}=1-y_{1}$.

A sample Maple code that computes the mixture volume and the molar volumes of twocomponent mixture is given below. The example is written for methane + nitrogen mixture for temperature $110 \mathrm{~K}$ and pressure $1 \mathrm{MPa}$ with methane mole fraction 0.75 .

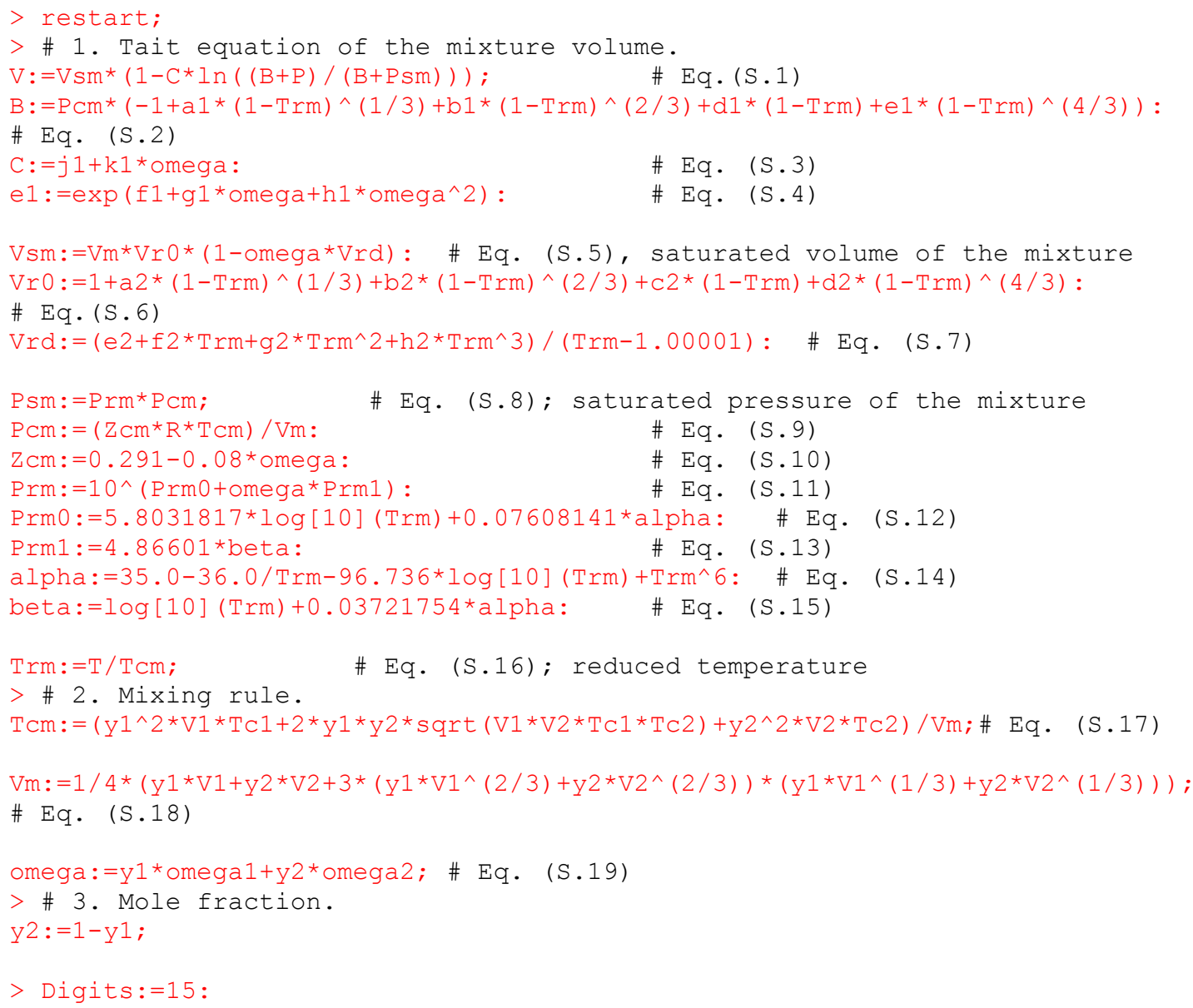




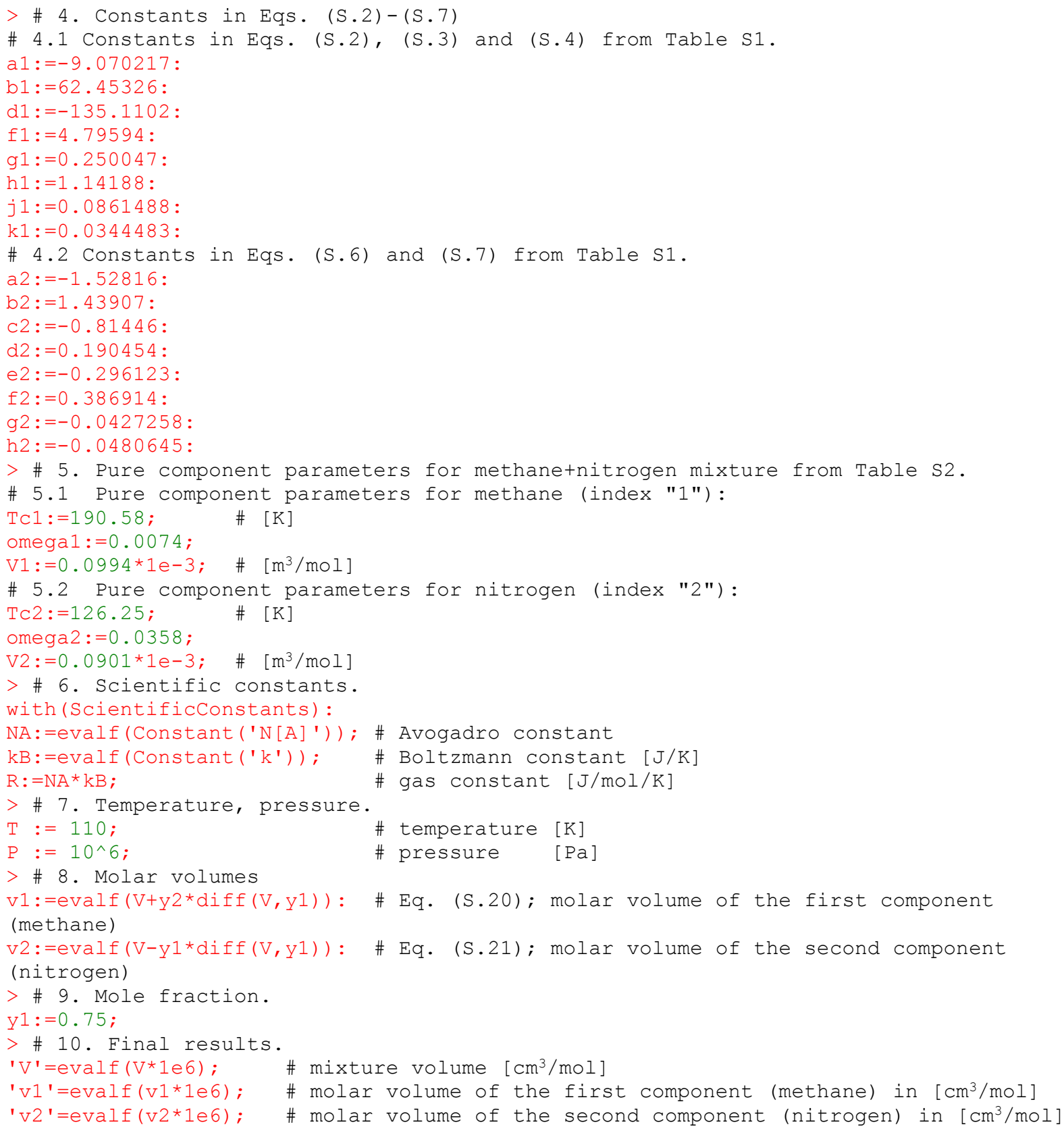

\section{S4. Radii of Cavitation}

Böttcher technique corresponds to substitution of experimentally measured dielectric constant into Onsager expression for the polarizability of a medium to obtain the respective cavitation radius, and analyse its dependence on structure, pressure, temperature etc. ${ }^{32}$ The technique is not possible to directly extend to mixtures as there one value of the dielectric permittivity cannot determine several cavitation radii. We therefore use an additional condition - the proportionality rule, Eq. (18). We substitute the experimental $\varepsilon$ from Refs. [27,28] into Eqs. (8)\&(9) and then solve the three equations (Eqs. (8), (9), and (18)) for $L_{Q}$, methane cavity radius $R_{\mathrm{cav}, 1}$, and nitrogen cavity radius $R_{\mathrm{cav}, 2}$. The results are presented in Figure $\mathbf{S 2}$. As one can see, the obtained $R_{\mathrm{cav}, i}$ using Böttcher procedure applied to mixtures are illogical. 
Furthermore, inconsistent results for $R_{\mathrm{cav}, i}$ are also obtained when this approach is applied to the classical Onsager model for liquid mixtures. We solve Eq. (6) together with the proportionality rule (18); this gives again $R_{\mathrm{cav}, 1}$ and $R_{\mathrm{cav}, 2}$. At some mixture composition the obtained cavity radii are in the order of 0.7-0.8 $\AA$, which is below the Curie point 1.3-1.4 $\AA$ (see Eq. (55)\&(56) in Ref. [9]).

These illogical results are most probably due to inaccuracy in the determined mixture density. If we use partial molar volumes that are only by $2 \%$ higher than the ones we computed, this would lead to a drop of the cavitation radius of methane to relatively reasonable values (e.g., the two points above $3.2 \AA$ in Figure S2 drop to about $2.5 \AA$ ).

In Figure $\mathbf{S 2}$ are presented also $R_{\mathrm{cav}, i}$ according Onsager relation Eq. (16) for $R_{\mathrm{cav}, i}\left(v_{i}\right)$ and the semi-empirical relation $R_{\mathrm{cav}, i}\left(\rho_{i}\right)$ from Eq. (17) (where $\rho_{i}=m_{i} / v_{i}$ ).
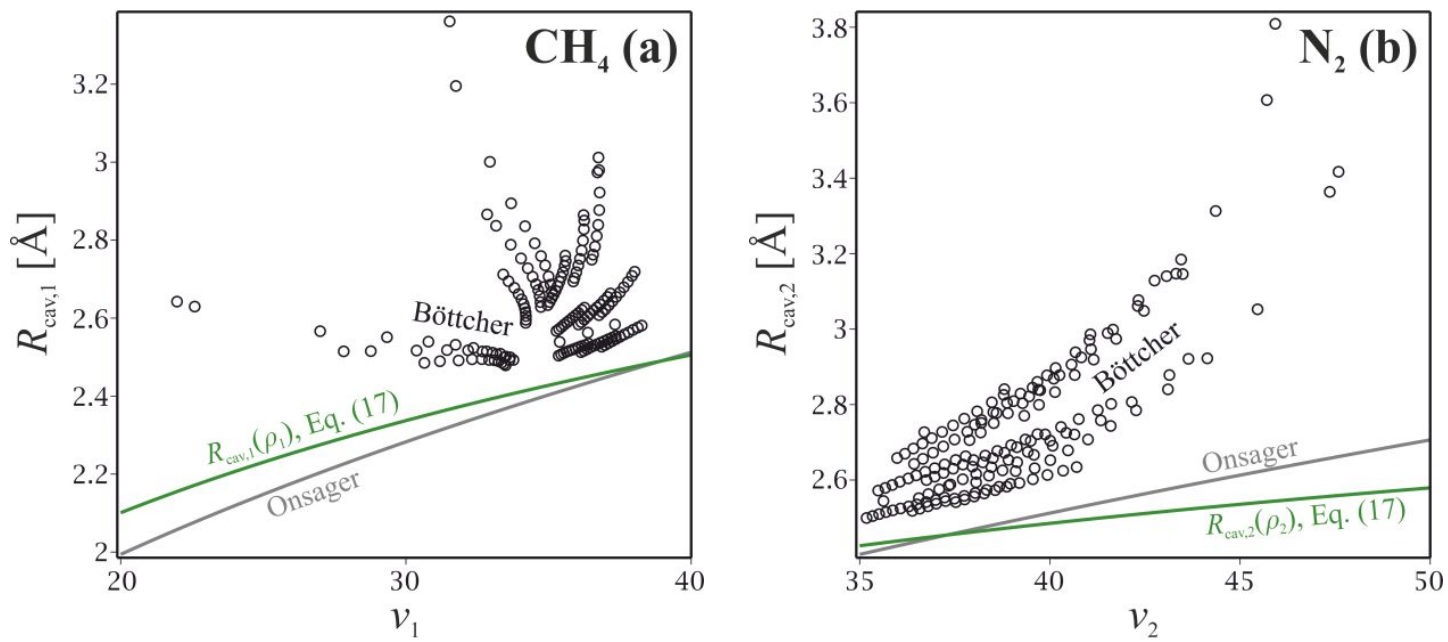

Figure S2. Methane cavity radius $R_{\mathrm{cav}, 1}$ (a) and nitrogen cavity radius $R_{\mathrm{cav}, 2}$ (b) as a function of the respective molar volumes $v_{1}$ and $v_{2}$. Unfilled circles are obtained using Böttcher technique. Gray curves represent Onsager relation Eq. (16) for $R_{\mathrm{cav}, i}\left(v_{i}\right)$; green curves are the semi-empirical relations $R_{\mathrm{cav}, i}\left(\rho_{i}\right)$ from Eq. (17).

\section{S5. Computational Procedures}

A Maple code that computes the quadrupolar length of $\mathrm{CH}_{4}+\mathrm{N}_{2}$ mixture using different computational procedures (as described in the main text) is supplemented below.

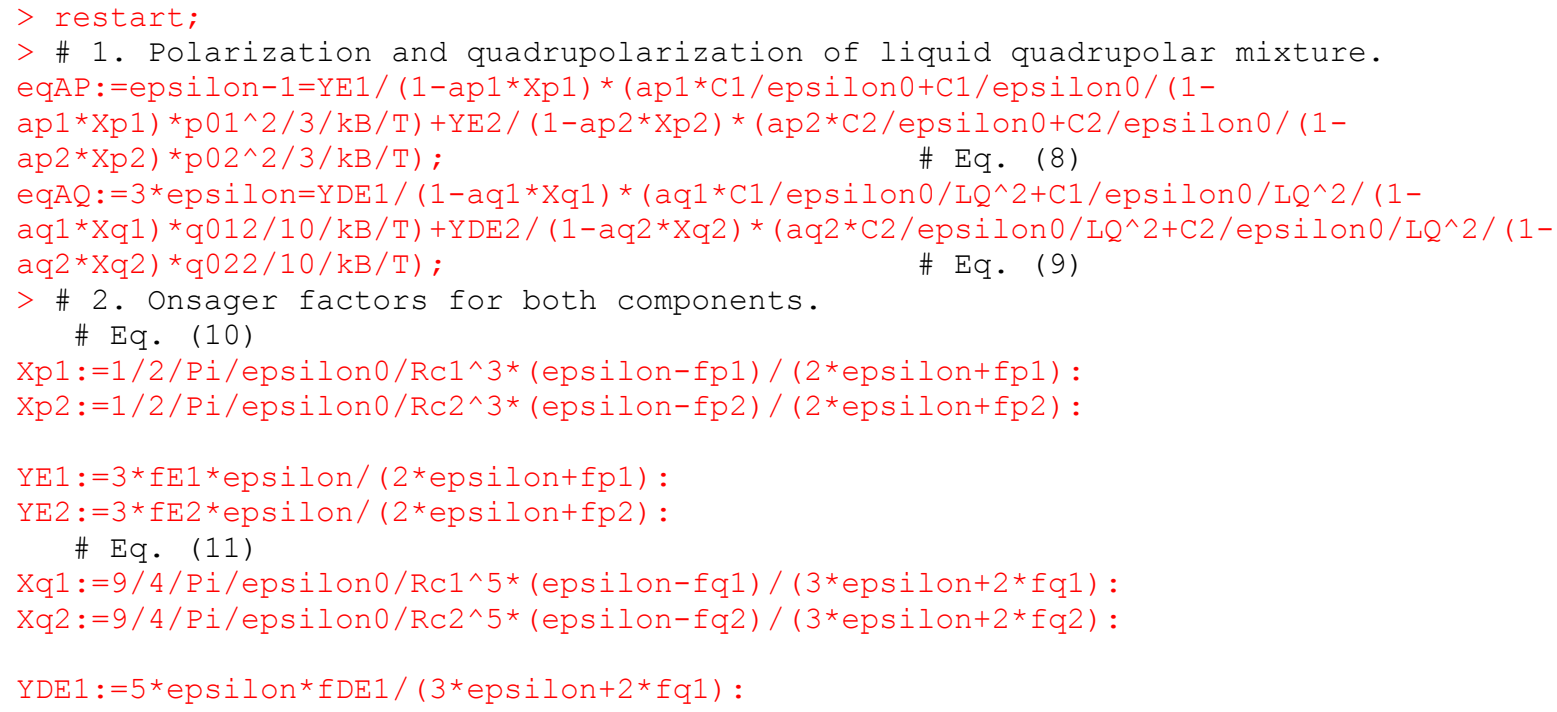




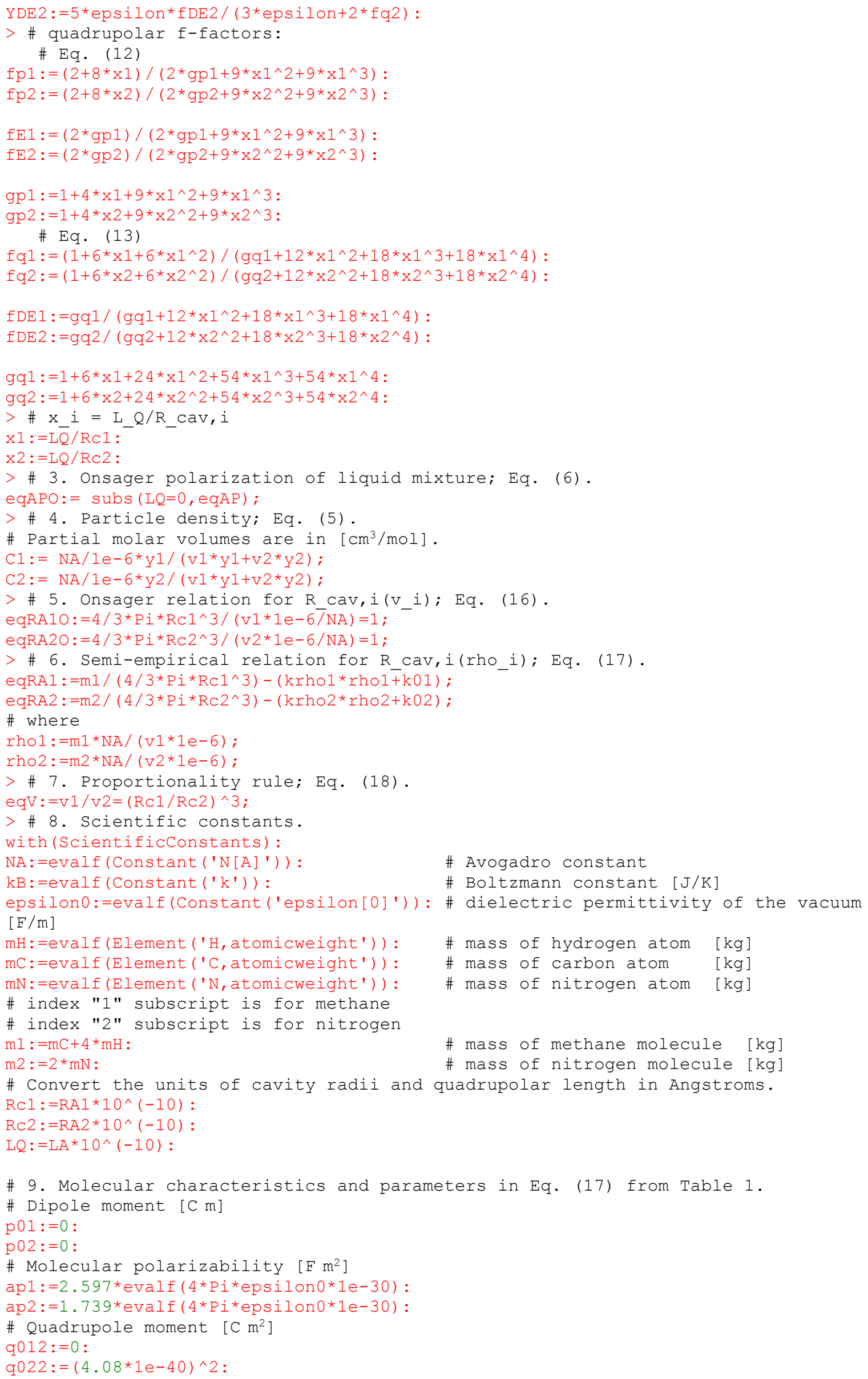


\# Molecular quadrupolarizability [ $\left.\mathrm{F} \mathrm{m}^{4}\right]$

aq1 $:=1.681 *$ evalf $(4 * \mathrm{Pi}$ epsilono*1e-50) :

aq2 $:=1.120 *$ evalf $(4 * P i * e p s i l o n 0 * 1 e-50):$

\# Parameters in Eq. (17)

$\mathrm{k} 01:=122.84: \quad$ \# $\left[\mathrm{kg} / \mathrm{m}^{3}\right]$

$\mathrm{k02}:=342.20: \quad \#\left[\mathrm{~kg} / \mathrm{m}^{3}\right]$

krho1:=0.7019: \# [/]

krho2: $=0.5445: \quad \#[/]$

$>$ \# 10. Additional parameters.

$\mathrm{T}:=100: \quad$ \# temperature $[\mathrm{K}]$

$\mathrm{P}:=20.01 \mathrm{e}$ : \# pressure [Pa]

\# Partial molar volumes, calculated using Hankinson-Thomson technique; Ref. [29, 30]

v1:=35.30689: \# methane partial molar volume $\left[\mathrm{cm}^{3} / \mathrm{mol}\right]$

v2:=35.50899: \# nitrogen partial molar volume $\left[\mathrm{cm}^{3} / \mathrm{mol}\right]$

\# Mole fractions

y1:=0.7462: \# methane mole fraction [/]

y2:=1-y1: \# nitrogen mole fraction [/]

\# Experimental dielectric constant of the mixture from Ref. [28]

epsilon_exp:=1.60552; \# [/]

$>$ \# 11. Computational procedures.

\# 11.1 Computational procedure (cpl) (Eqs. (8), (9), and (17)).

fsolve ( \{ subs ( [RA1=solve (eqRA1, RA1) [1], RA2=solve (eqRA2, RA2) [1] ], eqAP), subs ( [RA1=solve (eqRA1, RA1) [1], RA2=solve (eqRA2, RA2) [1] ], eqAQ) \}, $\{\mathrm{LA}=0.2$, epsilon=1..5 $\}) ;$

$>$ \# 11.2 Computational procedure (cp2) (Eqs. (8), (9), and (16)).

fsolve ( $\{$ subs ( [RA1=solve (eqRA10, RA1) [1], RA2=solve (eqRA2O, RA2) [1] ], eqAP), subs ( [RA1=solve (eqRA10, RA1) [1], RA2=solve (eqRA20, RA2) [1] ], eqAQ) \}, $\{\mathrm{LA}=0.2$, epsilon=1..5\});

> \# 11.3 Computational procedure (cpo) (Eqs. (6) and (16)) .

fsolve ( subs ( [RA1=solve (eqRA10,RA1) [1], RA2=solve (eqRA2O, RA2) [1] ], eqAPO), epsilon=1..5);

> \# 12. Minimal cavity radii according Curie point (see Eq. (55) \& (56) in Ref. [9]). R C1: $\max (\operatorname{evalf}((\operatorname{apl} /(4 * \mathrm{Pi} * \operatorname{epsilon0})) \wedge(1 / 3)) * 1 e 10$,

evalf $\left.\left((3 * a q 1 /(4 * \mathrm{Pi} * \operatorname{epsilon} 0))^{\wedge}(1 / 5)\right) \star 1 e 10\right)$;

R C2: $=\max (\operatorname{evalf}((\operatorname{ap} 2 /(4 * \mathrm{Pi} * \operatorname{epsilon} 0)) \wedge(1 / 3)) * 1$ e10,

evalf $\left.\left((3 * a q 2 /(4 * \mathrm{Pi} * \operatorname{epsilon} 0))^{\wedge}(1 / 5)\right) \star 1 e 10\right)$;

$>$ \# 13. Böttcher technique applied to mixtures.

fsolve ( $\{$ subs (epsilon=epsilon_exp, eqAP), subs (epsilon=epsilon_exp, eqAQ),

eqV\},

$\left.\left\{\mathrm{RA} 1=\mathrm{R} \_\mathrm{C} 1 \ldots 4, \mathrm{RA} 2=\mathrm{R} \_\mathrm{C} 2 \ldots 4, \mathrm{LA}=0.2\right\}\right) ;$ 